\title{
Therapeutic effects of $\alpha$-lipoic acid on bleomycin-induced pulmonary fibrosis in rats
}

\author{
RUI LIU ${ }^{1}$, KAZI MOKIM AHMED ${ }^{2}$, DANUPON NANTAJIT ${ }^{2}$, \\ FRANK S. ROSENTHAL ${ }^{2}$, CHUN-XUN HAI ${ }^{1}$ and JIAN JIAN LI ${ }^{2,3}$ \\ ${ }^{1}$ Department of Toxicology, Faculty of Preventive Medicine, Fourth Military Medical University, Xi'an, P.R. China; \\ ${ }^{2}$ School of Health Sciences, ${ }^{3}$ Purdue Cancer Research Center, Purdue University, West Lafayette, IN 47907, USA
}

Received February 12, 2007; Accepted March 16, 2007

\begin{abstract}
Pulmonary fibrosis (PF) is a major side effect of radiotherapy and chemotherapy. Recent clinical trials, unfortunately, have failed to identify any therapeutic agent which has the potential to reduce the consequences of this devastating condition. Reactive oxygen species and tissue remodeling regulators, such as metalloproteinases (MMPs) and their inhibitors (TIMPs), are thought to be involved in the development of PF. We investigated these factors to determine the protective effects of antioxidant $\alpha$-lipoic acid (LA) against antineoplastic agent bleomycin (BLM)induced oxidant lung toxicity in Sprague-Dawley rats. At different time intervals after BLM administration, pathological changes of the lung were analyzed with the measurement of total protein in bronchoalveolar lavage fluid (BALF), hydroxyproline (HYP) content and the level of three oxidative stress markers, i.e. malondialdehyde (MDA), the GSH/GSSG ratio, and total antioxidative capability (T-AOC). Also, the expression changes of MMP-1 and TIMP-1 were measured. At day 14 or 28 after BLM administration, protein content in BALF, and HYP, MDA and T-AOC contents of the lung increased significantly with a decreased GSH/GSSG ratio, implicating an increased efflux of GSSG from the lung and consumption of GSH. In contrast, treatment with LA
\end{abstract}

Correspondence to: Dr Jian Jian Li, School of Health Sciences, Purdue University, 550 Stadium Mall Drive, West Lafayette, IN 47907, USA

E-mail: jjli@purdue.edu

Abbreviations: BALF, bronchoalveolar lavage fluid; BLM, bleomycin; ECM, extracellular matrix; EDTA, Ethylenediaminetetraacetic acid; GSH, reduced glutathione; GSSG, oxidized glutathione; HYP, hydroxyproline; IPF, idiopathic pulmonary fibrosis; LA, $\alpha$-lipoic acid; MDA, malondialdehyde; MMP, matrix metalloprotease; NAC, N-acetylcysteine; PBS, phosphate-buffered saline; PF, pulmonary fibrosis; ROS, reactive oxygen species; RT$\mathrm{PCR}$, reverse transcriptase-polymerase chain reaction; TIMP, tissue inhibitor of metalloprotease; T-AOC, total antioxidant capacity

Key words: $\alpha$-lipoic acid, bleomycin, pulmonary fibrosis protected BLM-induced pulmonary injury by suppressing oxidative stress with the reduction of MDA, and the enhancement of the GSH/GSSG ratio and T-AOC. The BLM-stimulated symptoms of PF were relieved with significant reduction of HYP and total proteins in LA-treated rats. LA also ameliorated the MMP-1/TIMP-1 ratio. These results suggest that LA inhibits BLM-induced lung toxicity associated with oxidative damage. Therefore, antioxidant LA has a potential therapeutic effect in the prevention and alleviation of PF.

\section{Introduction}

Fibrosis is a reactive or reparative process characterized by the formation of excessive fibrous tissue. Lung fibrosis is associated with chronic inflammation characterized by the recruitment of macrophages, neutrophils, and lymphocytes in the airways (1). Accumulating evidence suggests that reactive oxygen species (ROS), e.g. superoxide, hydrogen peroxide, peroxynitrite, and hydroxyl radical are major mediators of lung inflammatory processes (2). Activated phagocytes release large amounts of ROS which induces tissue injury and inhibits tissue repair, leading to pulmonary fibrosis (PF) $(1,3,4)$. The main histological features of PF include lesions of the alveolar septa, fibroblast and myofibroblast proliferation in lung parenchyma, abnormal reepithelialization, and excessive extracellular matrix macromolecule deposition $(2,5,6)$. PF is a lethal interstitial lung disease of potential fatal prognosis and a severe post-radiotherapy or -chemotherapy side effect (7-9). Chronic oxidative stress has been linked with the radiation-induced injury of normal tissue including fibrosis (10). However, the elucidation of the molecular mechanism and development of a successful regimen for the prevention and treatment of $\mathrm{PF}$ remain an urgent challenge.

One of the clinically important causative agents of PF is bleomycin (BLM), a glycopeptide antibiotic used in the chemotherapy of different types of cancer which produces its antineoplastic effect by causing oxidant damage to DNA (11). BLM induces PF as an adverse effect since the cysteine hydrolase that inactivates BLM is relatively low in lung tissue. Intratracheal instillation of BLM into the lungs of various animal species causes alveolar cell damage, 
fibroblast proliferation and subsequent collagen deposition (11). The animal model of PF closely resembles the symptoms seen in patients (12), and it is a useful model with which to assess the effects of potential therapeutic agents, including antioxidant compounds. Previous studies have shown that antioxidants $\mathrm{N}$-acetylcysteine (NAC) and bilirubin protect rats against the tissue damage and PF induced by BLM $(13,14)$. Because these compounds can attenuate the oxidant burden in tissue, they may prevent the lung damage caused by ROS and subsequent fibrosis.

Matrix metalloproteinases (MMPs), a family of extracellular and cell surface-associated proteinases for tissue remodeling and fibrosis, are associated with inflammatory conditions $(15,16)$. MMPs and their specific inhibitors, TIMPs (tissue inhibitor of metalloproteinases), are the hallmark of this fibrogenic microenvironment. MMP-1 (also known as collagenase-1), produced by fibroblasts, endothelial and epithelial cells and by cells of macrophage lineage, preferentially degrades type III collagen. Increased MMP-1 expression by epithelial cells overlying intra-alveolar fibrosis has been identified in lung samples from IPF (idiopathic pulmonary fibrosis) patients (17). These results suggest that MMP-1 may contribute to the collagenase activity in BALF (bronchoalveolar lavage fluid) and plays an essential role in PF. A 'nondegrading microenvironment' caused by the induction of TIMP-1 levels in lung tissue by various agents including BLM, which in turn lowers the MMP/TIMP ratio, retains microenvironments and induces fibrogenicity (5,18-20). However, the exact molecular mechanisms by which the MMP/TIMP ratio contributes to the PF phenotype remain elusive.

The present study evaluated the effects of antioxidant $\alpha$-lipoic acid (LA) in a rat model of lung injury induced by endotracheal instillation of BLM. Markers of PF and oxidant stress, and the MMP-1/TIMP-1 ratio were measured 7, 14, and 28 days post-BLM exposure. PF was assessed by a semiquantitative histological score and total protein in BALF, and lung hydroxyproline was measured as a marker of collagen deposition. MDA content, the GSH/GSSG ratio, and T-AOC were measured as oxidative stress markers. Our data show that LA significantly ameliorates all these markers of lung damage at 14 and/or 28 days of BLM administration, suggesting that LA is a potential inhibitor for ROS-mediated lung toxicity and PF.

\section{Materials and methods}

Materials and animals. Bleomycin A5 hydrochloride (BLM), a total antioxidative capacity (T-AOC) kit and thiobarbituric acid (TBA) were obtained from Taihe Pharmaceutical Co. (Tianjin, P.R. China), Jiancheng Bioengineering Institute (Nanjing, P.R. China) and Merck (New Jersey, USA), respectively. Glutathione (GSH), nitroblue tetrazolium (NBT), 1,1,3,3-tetraetoxypropane and 5',5'-dithiobis-2nitrobenzoic acid (DTNB) were purchased from Sigma (St. Louis, MO, USA). Other chemicals and reagents were purchased from standard suppliers.

A total number of 45 male Sprague-Dawley rats (12 weeks old), weighing 250-300 g at the start of the experiments, were housed in a specific cage with a 12-h light/dark cycle and free access to water and food ad libitum. Rats were randomly divided into three groups of fifteen animals each (control, BLM, and BLM+LA). All animals were instilled intra-tracheally by the same volume, $0.1 \mathrm{ml} / \mathrm{kg}$. Control animals received saline and other groups were administered bleomycin hydrochloride ( $5 \mathrm{mg} / \mathrm{kg}$ body weight). According to the results from a preliminary dose-response study, BLM+LA rats were intraperitoneally injected with $20 \mathrm{mg} / \mathrm{kg}$ body weight LA ( $\alpha$-lipoic acid) individually once daily after an intratracheal injection of BLM solution. Other groups received the same volume of intraperitoneal saline. The rats were sacrificed at 7,14 and 28 days after BLM instillation. Pulmonary fibrosis (PF) was assessed by lung histology, hydroxyproline (HYP) content, oxidative stress marker levels and total protein in bronchoalveolar lavage fluid (BALF).

Histological assessment. To elucidate the histopathologic changes associated with BLM-induced lung fibrosis and the efficacy of LA, left lungs from control (saline-treated), BLM and BLM+LA groups were collected 7, 14, and 28 days after BLM instillation. The lung sections were fixed in $4 \%$ paraformaldehyde for $24 \mathrm{~h}$ and then processed for paraffin embedding. Sections of lung were stained with haematoxylin and eosin and Masson's trichrome to assess the degree of fibrosis. Each successive field was individually assessed for extent and severity of interstitial fibrosis using the semiquantitative grading system as previously described (21). The entire lung section was reviewed at a magnification of $\mathrm{x} 100$. For each lung section 30-35 microscopic fields were needed to review the section. Criteria for grading PF were as follows: grade $0=$ normal lung; grade $1=$ minimal fibrous thickening of alveolar or bronchial walls; grade $2-3=$ moderate thickening of walls without obvious damage to lung architecture; grade 4-5 = increased fibrosis with definite damage to lung architecture and formation of fibrous bands or small fibrous mass; grade 6-7 = severe distortion of structure and large fibrous areas; and grade $8=$ total fibrous obliteration of the field. The mean score of all fields was taken as the fibrosis score of that lung section.

Protein content in BALF. Rats were anaesthetized with an i.p. administration of sodium thiopental $(70 \mathrm{mg} / \mathrm{kg})$ and sacrificed. BALF was collected by washing the right lung three times with 2-ml aliquots of saline through a tracheal cannula. Typically, total fluid recovery exceeded $85 \%$, and the percentages of fluid recovered did not significantly differ among experimental groups, i.e. BLM and BLM+LA rats. Total protein concentration in cell-free BALF supernatants was determined using Lowry's method and expressed as grams of protein per liter of BALF $(\mathrm{g} / \mathrm{l})$.

Hydroxyproline measurement. Hydroxyproline (HYP) is a major component of the protein collagen, and it permits the sharp twisting of the collagen helix. Therefore, the measurement of this protein is an efficient index of fibrosis. Lung HYP content was measured spectrophotometrically as outlined by Nagase and Woessner (22). Briefly, lung tissue was homogenized and then hydrolyzed in $6 \mathrm{~N} \mathrm{HCl}$ for $18 \mathrm{~h}$ at $110^{\circ} \mathrm{C}$. The hydrolysate was then neutralized with $2.5 \mathrm{M}$ $\mathrm{NaOH}$. Aliquots $(2 \mathrm{ml})$ were mixed with citrate buffer $(0.25 \mathrm{M}$, 
$\mathrm{pH}$ 6.0), and the reaction was terminated by $1.0 \mathrm{ml}$ chloramine $\mathrm{T}(0.05 \mathrm{M})$, and $1.0 \mathrm{ml}$ perchloric acid $(3.15 \mathrm{M})$. The samples were mixed with $1.0 \mathrm{ml}$ dimethylaminobenzaldehyde $(100 \mathrm{~g} / \mathrm{l})$ and incubated for $10 \mathrm{~min}$ at $80^{\circ} \mathrm{C}$. The colored products were read for absorbance at $560 \mathrm{~nm}$ in a spectrophotometer, and the results were expressed as micrograms of HYP per lung.

Analysis of lung tissue oxidants and antioxidants. Lung MDA (malondialdehyde) levels were determined by Yagi's method (23), which is based on the reaction of MDA with thiobarbituric acid at $100^{\circ} \mathrm{C}$. The total protein concentration was determined using CBB (Coomassie brilliant blue G250) staining, and the results were expressed as micromole per gram wet tissue protein of lung ( $\mu \mathrm{mol} / \mathrm{g}$ protein). Reduced glutathione (GSH) in lung tissue was measured fluorometrically as described previously $(24,25)$. Briefly, a $200-\mu 1$ BALF sample was mixed with an equal amount of $10 \%$ TCA and then centrifuged at $5000 \mathrm{x}$ g for $10 \mathrm{~min}$. An aliquot $(100 \mu \mathrm{l})$ of the supernatants was mixed with $100 \mu 1$ formaldehyde, $3.6 \mathrm{ml}$ of $0.1 \mathrm{M}$ phosphate buffer ( $\mathrm{pH} 8.3,5 \mathrm{mM}$ EDTA), $200 \mu 1$ $0.1 \%$ o-phthalaldehyde (OPT) followed by incubation at room temperature for $40 \mathrm{~min}$. The GSH level in the mixture was measured in a fluorospectrophotometer at $553 \mathrm{~nm}$ with excitation at $515 \mathrm{~nm}$. Commercially procured GSH (Sigma) was used to determine a standard curve. The results were expressed in nanomoles GSH per milligram protein. The same method was used to detect GSSG except $0.1 \mathrm{M}$ phosphate buffer (pH 8.3, 5 mM EDTA) was substituted by $0.1 \mathrm{M}$ $\mathrm{NaOH}$. The total antioxidant capacity (T-AOC) of plasma and supernatant from lung tissue was measured using an assay kit (Jiancheng Bioengineering Institute, Nanjing, P.R. China) according to the manufacturer's instructions. The protocols did not distinguish between lipid- and water-soluble antioxidants, but provided an estimation of global antioxidant capacity. All samples were assayed in duplicate and the results were the averaged values.

RNA preparation and RT-PCR of MMP-1 and TIMP-1. Total RNA was isolated from right lung homogenates with TRIzol reagent (Invitrogen, Carlsbad, CA) following the manufacturer's instructions. Analysis of MMP-1 and TIMP-1 mRNA expression was made by the RT-PCR method. Using total RNA $(2 \mu \mathrm{g})$, RT-PCR for MMP-1, TIMP-1 and B-actin control was performed using Gene Amp PCR System 9700 (Perkin Elmer Applied Biosystems, Foster City, CA). The forward and reverse primers were: MMP-1 (forward 5'-GCC ATT ACC AGT CTC CGA GGA-3' and reverse 5'-GGA ATT TGT TGG CAT GAC TCT CAC-3'); TIMP-1 (forward 5'-CCC AAC CCA CCC ACA GAC-3' and reverse 5'-GGC CCG CGA AGA GAA ACT-3'); and ß-actin (forward 5'-TCA CCC ACA CTG TGC CCA TCT A-3' and reverse 5'-CAT CGG AAC CGC TCA TTG CCG ATA G-3'). The PCR (15- $\mu 1$ reaction mixture) was carried out using the following thermal cycles: $94^{\circ} \mathrm{C}$ for $5 \mathrm{~min}$, and $94^{\circ} \mathrm{C}$ for $30 \mathrm{sec}, 62^{\circ} \mathrm{C}$ for $45 \mathrm{sec}$ and $68^{\circ} \mathrm{C}$ for $45 \mathrm{sec}$ for 30 cycles, followed by a final elongation at $72^{\circ} \mathrm{C}$ for $7 \mathrm{~min}$. The amplified PCR products (5 $\mu \mathrm{l}$ ) were separated by electrophoresis on a $1.2 \%$ agarose gel containing ethidium bromide, and were photographed under UV light (Bio-Rad Gel system). The intensity of each cDNA band was quantitated and normalized to the corresponding $\beta$-actin cDNA band intensity (internal control) using a Bio-Rad Gel Doc 2000 System. RT-PCR and gel electrophoresis were performed in triplicate for all specimens.

Immunohistochemical detection of MMP-1 and TIMP-1. Five-micron lung sections were deparaffinized and then treated with $0.2 \%$ hydrogen peroxide in $0.1 \mathrm{M}$ PBS for $30 \mathrm{~min}$ to block endogenous peroxidase. Following three washings with PBS, the sections were exposed to $1.5 \%$ normal goat serum for $30 \mathrm{~min}$ to block nonspecific binding. Sections were then washed in PBS and incubated with primary antisera including rabbit anti-MMP-1, -TIMP-1, and a negative control antibody (1:200 dilutions; Boster, P.R. China) overnight at $4^{\circ} \mathrm{C}$. This was followed by three washings in PBS, and incubation with a biotinylated anti-rabbit IgG (secondary antibody; 1:200 dilutions; Boster) for $30 \mathrm{~min}$ at $37^{\circ} \mathrm{C}$ in a solution containing the avidin-biotin peroxidase complex (1:100 dilutions; ABC kit, Boster) for a further $30 \mathrm{~min}$. The reaction was developed with diaminobenzidine-hydrogen peroxide solution $\left(0.005 \% 3,3^{\prime}\right.$-diaminobenzidine and $0.1 \%$ hydrogen peroxide in $0.1 \mathrm{M}$ PBS). Before mounting, the sections were counterstained with haematoxylin and eosin.

Statistical analysis. Data for control and experimental groups were expressed as means \pm SEM of n (number of observations) experiments. Statistical analysis was carried out by analysis of variance (ANOVA) followed by appropriate post hoc tests including multiple comparison tests (LSD) or the nonparametric Mann-Whitney U test. All analyses were made using the SPSS version 11.0 statistical software package (SPSS, Inc., Chicago, IL), and a probability (P) value $<0.05$ was considered significant.

\section{Results}

\section{Assessment of pulmonary fibrosis}

Histopathology. Haematoxylin and eosin- and Masson's trichrome-stained lung sections were examined by light microscopy to determine whether BLM-induced pulmonary fibrosis was decreased by treatment with LA. Lung sections from control rats were histologically normal at day 7, 14 and 28 (Fig. 1A and D, and not shown). At day 7 post-BLM, severe edema, large amounts of inflammatory cells (including neutrophils and lymphocytes) in both alveoli and interstitium, and damaged endothelia and alveolar epithelia cells were observed. Treatment with LA resulted in significant attenuation of these histopathologic findings (not shown). At day 14 post-BLM, foci of collagen deposition and fewer inflammatory changes were observed (Fig. 1B and E). Marked histopathologic changes, such as large fibrous areas, collapsed alveolar spaces, and traction bronchiectasis in the subpleural and peribronchial regions, were seen at day 28 (not shown). In contrast, although fibrotic lesions were observed in lung sections of the BLM+LA group at day 14 or 28 (Fig. 1C and F, and not shown), the extent of fibrosis was markedly less severe compared with that in the BLM group. To confirm the effects of LA on the histopathology of BLM-induced lung injury and fibrosis, the overall grades of lung fibrosis were estimated by numerical scoring after 14 days. Relative to the BLM group, the scores of fibrosis in the lung sections of the 


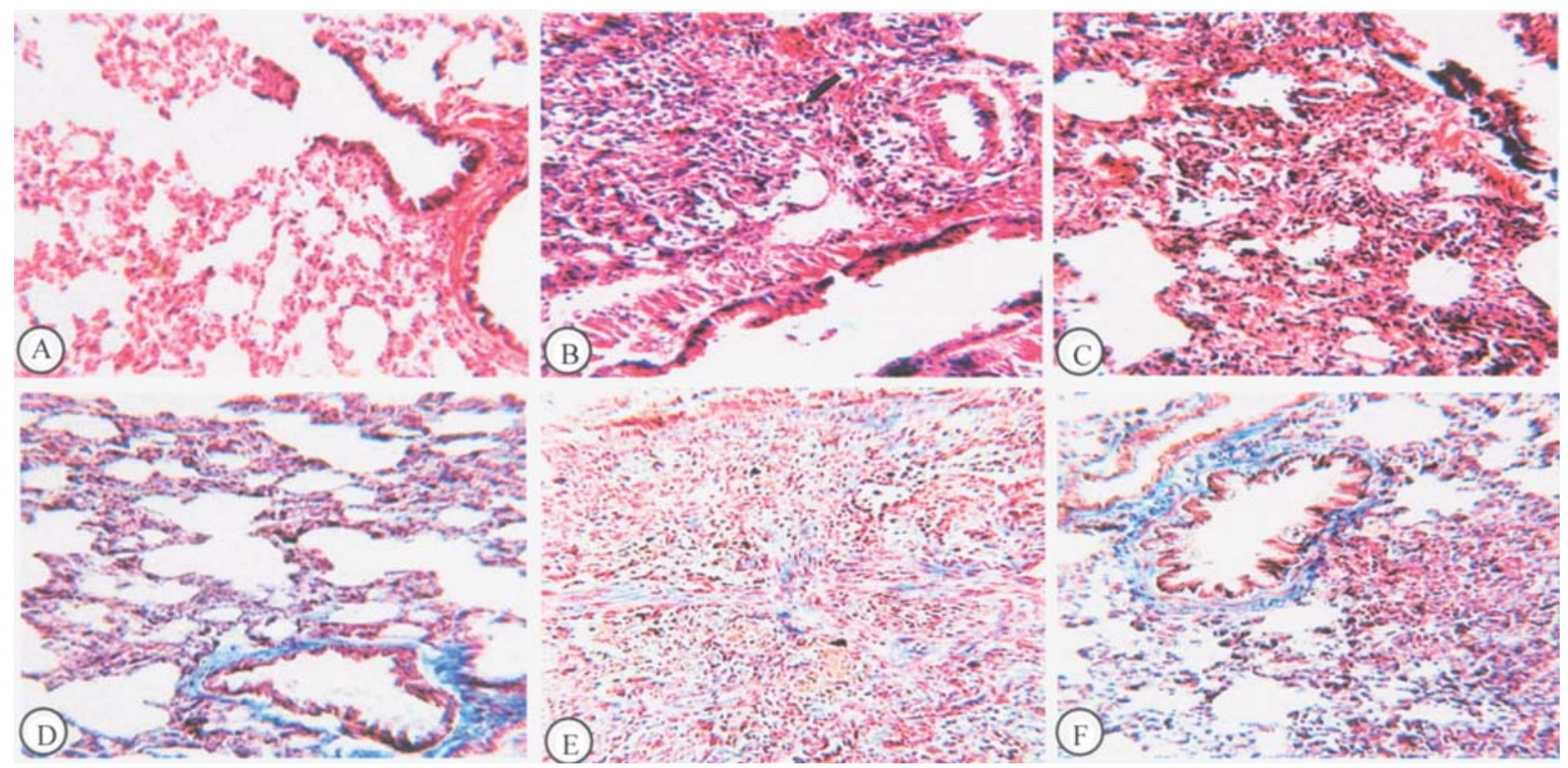

Figure 1. Representative photomicrographs of lung histology in the control (saline-treated; A and D), BLM (B and E) and BLM+LA (C and F) groups. Lung tissues were obtained 14 days post-treatment, and stained with haematoxylin and eosin (A-C) and Masson's trichrome (D-F) to evaluate histological changes of the lungs; magnification, x100. A and D, normal appearance of lung histology in the control group. B and E, BLM produced a peribronchial interstitial infiltration with inflammatory cells, oedema and fibrosis. C and F, reduction of BLM-induced histological changes by LA.

BLM+LA group were significantly decreased. The grades of fibrosis in the three groups are presented in Table I.

Protein content in BALF and lung hydroxyproline measurement. Increased protein concentration in BALF is an important marker of alveolar edema in the acute phase of the fibrogenetic process. At day 7, the protein content was similar in both BLM alone and BLM+LA-treated rats, although it was increased markedly when compared with the control group (Fig. 2A). Also, protein in BALF was similar in the control rats after 28 days compared to the LA-treated rats with or without BLM administration. In contrast to 7- or 28day treatment, at day 14 LA treatment led to a significant reduction in the BLM-induced increase in protein content in alveoli, suggesting that LA has a great potential for ameliorating lung edema.

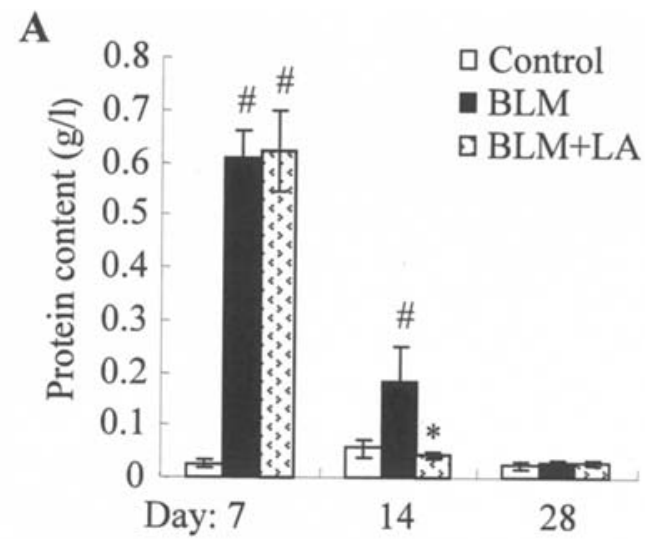

Table I. Grades of pulmonary fibrosis 14 days post-treatment.

\begin{tabular}{lc}
\hline Groups & Grade of fibrosis \\
\hline Control & $0.89 \pm 0.08$ \\
BLM & $4.52 \pm 0.34^{\mathrm{a}}$ \\
BLM+LA & $3.46 \pm 0.21^{\mathrm{ab}}$ \\
\hline
\end{tabular}

${ }^{\mathrm{a}} \mathrm{P}<0.05$ versus control; ${ }^{\mathrm{b}} \mathrm{P}<0.05$ versus $\mathrm{BLM}$.

Lung HYP (hydroxyproline) concentration reflects collagen deposition in lungs. Therefore, pulmonary fibrosis can be quantified by measuring HYP content in lungs as an index of collagen accumulation. Fig. 2B shows the effect of

B

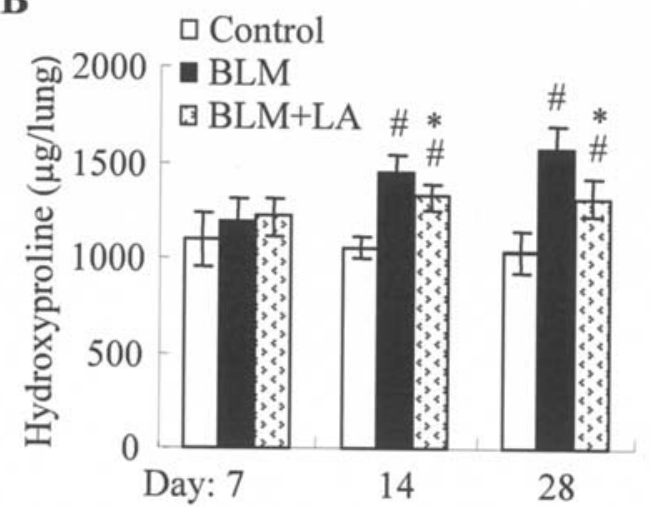

Figure 2. Inhibition of BLM-induced total proteins and hydroxyproline by LA. (A) Total proteins in BALF were measured using Lowry's method at 7, 14 and 28 days in rats instilled with BLM intratracheally with or without LA treatment. (B) Lung HYP content was measured spectrophotometrically at 7, 14 and 28 days in the BLM and BLM+LA groups. Data are the mean \pm SEM of three independent observations. ${ }^{*} \mathrm{P}<0.05$ from $\mathrm{BLM}$; ${ }^{\text {"P }}<0.05$ from control. 
A

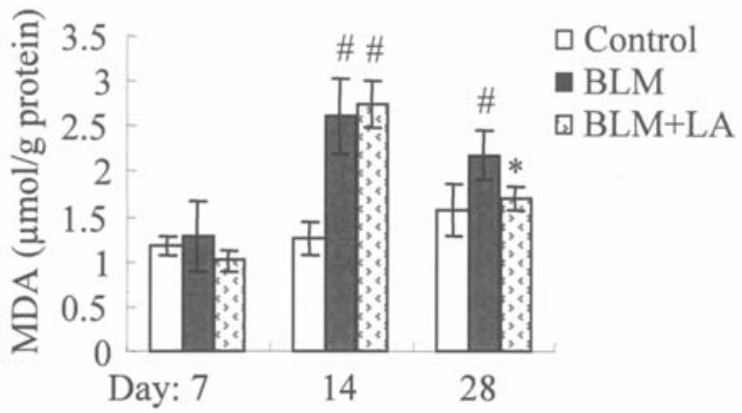

B

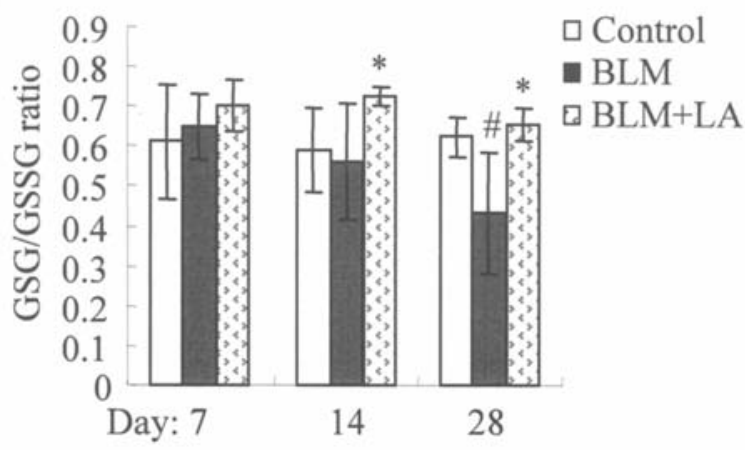

C

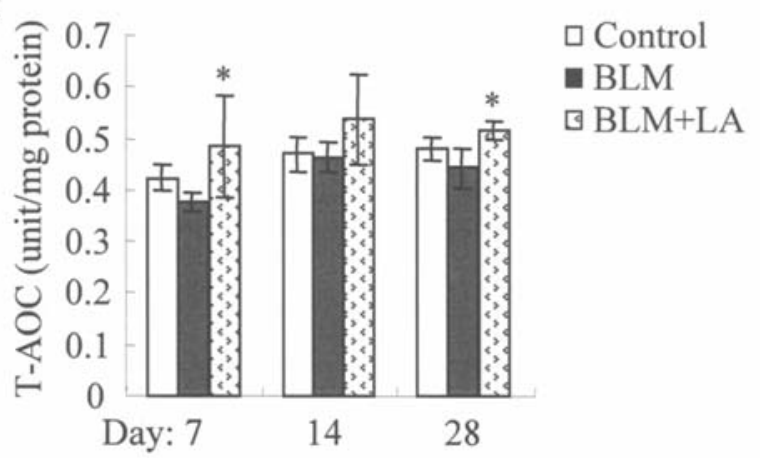

Figure 3. Effect of LA in BLM-modulated malondialdehyde (MDA), the GSH/GSSG ratio, and total antioxidant capacity (T-AOC) at day 7, 14 and 28 after BLM administration. (A) Reduction of MDA levels in lung tissue after 28 days of BLM administration in rats treated with intraperitoneal LA. (B) Enhancement of BLM-induced decreases in the GSH/GSSG ratio at day 14 and 28 by LA. The GSH and GSSG contents of the lung in the BLM and BLM+LA groups were measured as described in Materials and methods to determine the GSH/GSSG ratio. (C) Induction of T-AOC levels after 7 and 28 days post-BLM in the LA-treated group. Data are presented as the mean \pm SEM. ${ }^{*} \mathrm{P}<0.05$ versus $\mathrm{BLM} ;{ }^{*} \mathrm{P}<0.05$ versus control.

LA on the HYP content of lung homogenates at 7, 14, and 28 days following BLM intratracheal administration. The HYP level was similar at day 7 post-BLM with or without LA treatment. However, at day 14 or 28 , it was significantly higher in rats exposed to BLM alone. In contrast, treatment with LA led to a significant reduction in the HYP content $(\mathrm{P}<0.05)$, indicating the beneficial effects of LA in protecting against pulmonary fibrosis induced by BLM.

\section{Analysis of oxidative stress markers}

MDA content. Oxidative stress plays an important role in the development of fibrotic responses in the lung. MDA (malondialdehyde) is a highly reactive three-carbon dialdehyde produced as a by-product of polyunsaturated fatty acid peroxidation and arachidonic acid metabolism. The MDA level, an index of oxidative stress, was increased in lung tissue at day 14 or 28 , but not 7 post-BLM treatment (Fig. 3A) when compared with that of the control rats. LA significantly prevented increases in the lung tissue MDA content produced by BLM at day $28(\mathrm{P}<0.05)$, indicating the crucial role of LA in reducing BLM-mediated oxidative stress.

GSH/GSSG ratio. The GSH/GSSG ratio is an index of the tissue redox state. We measured GSH (reduced glutathione) and GSSG (oxidized glutathione) in lung tissue at day 7, 14 and 28 post-BLM with or without LA treatment. As shown in Fig. 3B, a decrease in the GSH/GSSG ratio (a decrease in GSH accompanied by an increase in GSSG) was observed in rats instilled with BLM. These changes were significantly reversed in the rats treated with intraperitoneal LA $(\mathrm{P}<0.05)$. Therefore, treatment with LA resulted in a significant enhancement in the GSH/GSSG ratio in the lung compared with that in the BLM group.

T-AOC measurement. We further investigated the redox status by measurement of total antioxidant capacity (T-AOC) in lung tissue at day 7,14 and 28 post-BLM with or without LA treatment. Although the T-AOC of the lung was slightly reduced following BLM exposure, it was significantly increased at day 7 and 28 by treatment with $\mathrm{LA}(\mathrm{P}<0.05$; Fig. 3C). Together with Fig. 3A and B, this result indicates that LA treatment markedly ameliorated BLM-induced alterations in oxidative damage and antioxidative enzyme activities

MMP-1 and TIMP-1 expression

$M M P-1$ and TIMP-1 gene $m R N A$ analysis by RT-PCR in rat lung tissue. Metalloproteinases (MMPs) play a crucial role in the fibrogenic process (16), and MMP activity is closely regulated through endogenous inhibitors, mainly through TIMPs (22). The abnormal expression of MMPs and/or TIMPs has a role in human lung fibrosis $(5,26)$. To investigate whether the reduction of BLM-induced lung fibrosis by LA (Fig. 1; Table I) was caused by altered degradation of collagen, we analyzed mRNA for MMP-1 and TIMP-1 by the RT-PCR method. Fig. 4 shows that both MMP-1 and TIMP-1 mRNA levels were raised at day 7 post-BLM and -BLM+LA compared to the control rats. The MMP-1 mRNA level was decreased at day 14 but increased at day 28 by BLM exposure. However, MMP-1 expression remained constant at day 7 or 14 in rats exposed to BLM and LA (Fig. 4A and B; left panel). The increase in TIMP-1 mRNA at day 7 persisted for 28 days following BLM exposure (Fig. 4A and B; right panel). In contrast, relative to the control, the enhanced TIMP-1 mRNA at day 7 was found reduced at day 14 or 28 by combined treatment of BLM and LA. These results indicate that bleomycin injury led to a significant increase in TIMP-1 at all time points, while LA could lower its expression at day 14 or 28. Taken together, as described below (Fig. 5), the differential levels of MMP-1 and TIMP-1 mRNA indicate an altered MMP-1/TIMP-1 ratio in rats treated with BLM+LA compared to BLM alone. 
A

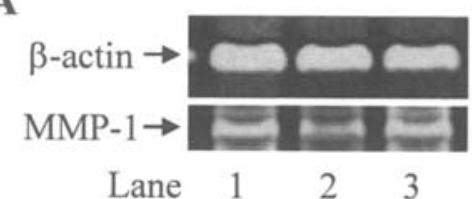

B

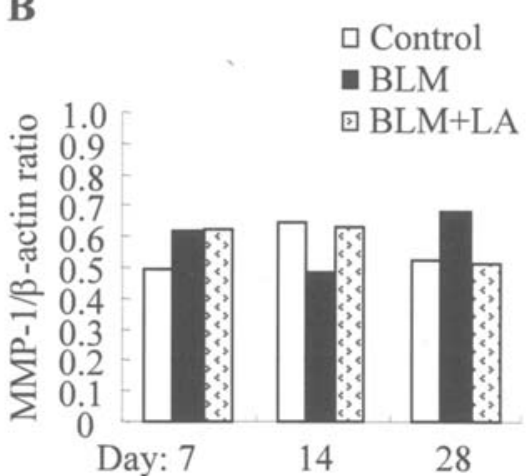

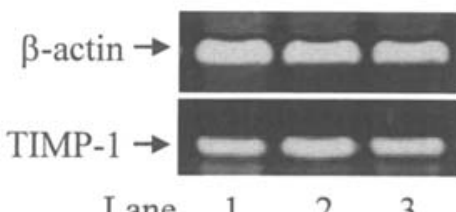

Lane $1 \quad 2 \quad 3$

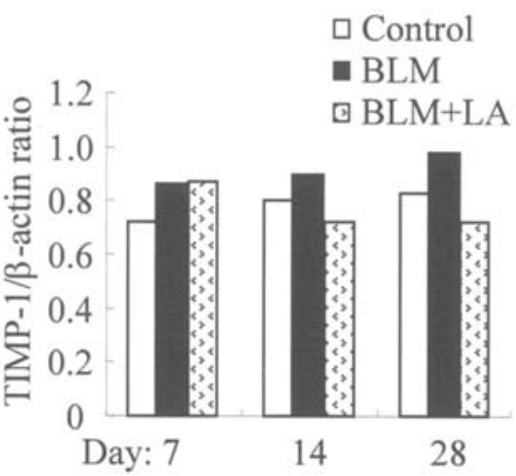

Figure 4. RT-PCR analysis of mRNA expression levels of MMP-1 and TIMP-1. (A) Total RNA was isolated from the lung tissue of the control, BLM and BLM+LA groups for RT-PCR analysis at day 7, 14 and 28 (data not shown). Lanes 1, 2 and 3 correspond to control, BLM and BLM+LA, respectively. (B) The relative MMP-1 (left panel) and TIMP-1 (right panel) mRNA levels, at day 7, 14 and 28 in each group as indicated were estimated using densitometry and were normalized to control $\beta$-actin. Note: MMP-1 expression in lane 2 (A; left lower panel) was low when compared with lane 3; even the ratio showed no difference (B, left panel).

A
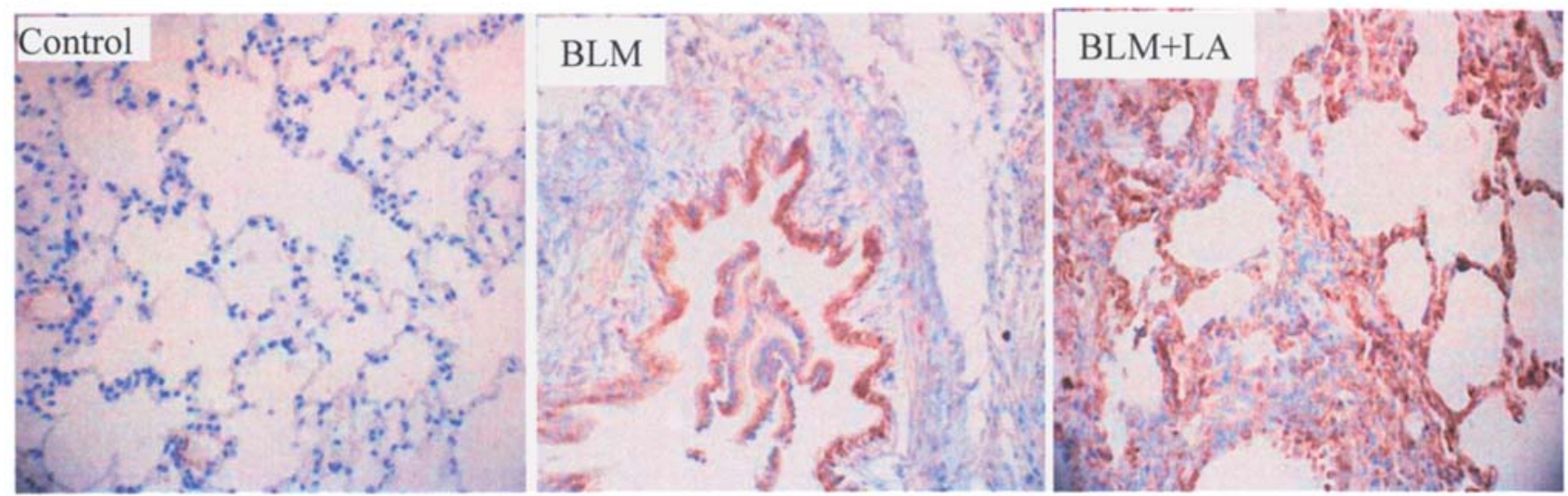

\section{B}

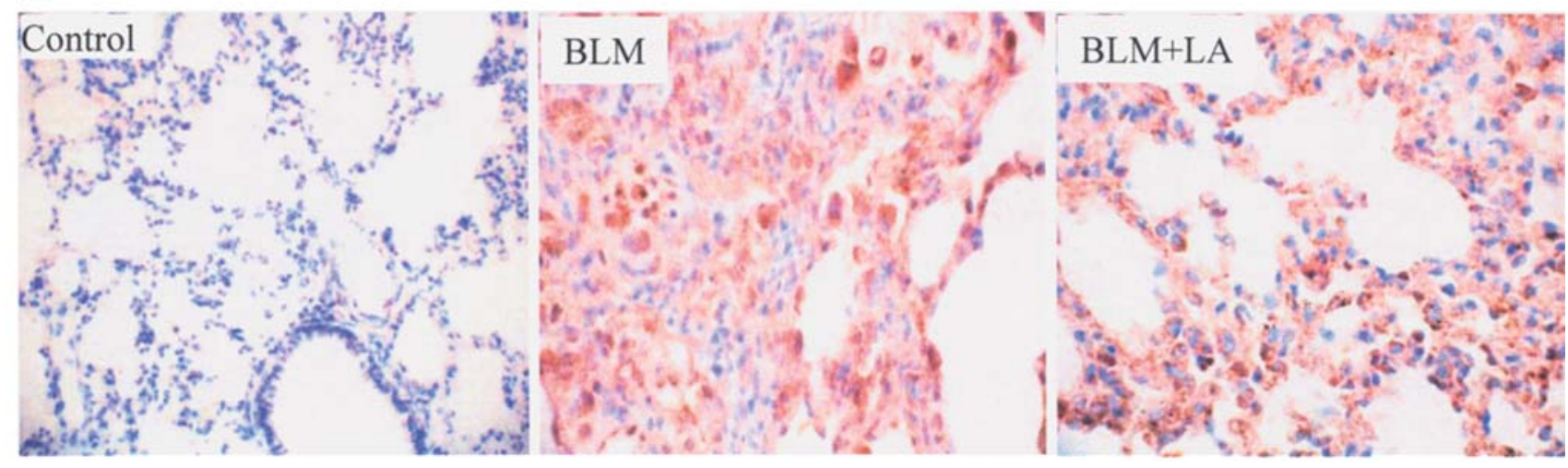

Figure 5. Immunohistochemical analysis of MMP-1 and TIMP-1. Lung sections were obtained at day 14 from control rats and from rats exposed to BLM with or without LA treatment. (A) Induction of MMP-1 expression in BLM-treated rats, which was further enhanced slightly in BLM+LA rats. Lung sections were stained with anti-MMP-1 antibody followed by incubation with avidin-biotin peroxidase complex. The reaction was developed with diaminobenzidinehydrogen peroxide solution. The immunohistochemical localization of MMP-1 appears as dark-brown staining. There is a low staining level in control lungs (original magnification x40). (B) Reduction of BLM-induced TIMP-1 expression by LA. Lung sections were stained with antibody to TIMP-1 and then incubated with the avidin-biotin complex. The reaction was developed with diaminobenzidine-hydrogen peroxide solution. The immunohistochemical localization of TIMP-1 appears as dark-brown staining. There is a low staining level in control lungs (original magnification $\mathrm{x} 40$ ). 
$\alpha$-lipoic acid modulates BLM-induced MMP-1 and TIMP-1 protein expression. A decrease in the MMP/TIMP ratio is believed to worsen lung fibrosis. For instance, significant increases in both MMP-9 and TIMP-1 expression, i.e. a decrease in the MMP-9/TIMP-1 ratio in the WT mouse lungs were detected after bleomycin treatment (27). The fibrogenetic cytokines (e.g. TGF-ß) reduce collagen degradation by downregulation of MMPs and upregulation of TIMPs $(28,29)$. In this study, lung sections from the control rats showed a small degree of immunostaining for MMP-1 (Fig. 5A) and TIMP-1 (Fig. 5B). An increase in staining (dark-brown) for MMP-1 was noticed at 14 days post-bleomycin exposure, and LA treatment slightly enhanced MMP-1 immunostaining (Fig. 5A). Compared to the control rats, the immunostaining for MMP-1 inhibitor TIMP-1 was severely increased in rats injected with bleomycin. The increased level of dark-brown TIMP-1 staining was significantly reduced by LA (Fig. 5B). These results indicate that LA ameliorates the MMP-1/ TIMP-1 ratio, which indicates a potential inhibitory role of LA in BLM-induced pulmonary fibrosis in rats.

\section{Discussion}

Pulmonary fibrosis (PF) is a severe side effect of anti-cancer therapy, characterized by an altered cellular composition of the alveolar region with excessive deposition of collagen. Lung inflammation is thought to be a major underlying component of a wide variety of pulmonary fibroproliferative disorders. Although current medical therapies have mainly focused on anti-inflammation with glucocorticoid and some cytotoxin-like medicine, an ideal therapeutic strategy for PF in the clinic has yet to be established. The present study examined, for the first time in vivo, the potential protective effects of $\alpha$-lipoic acid (LA) in an animal model of bleomycin (BLM)-induced PF. LA is a thiol-replenishing and redox modulating agent, which acts as an effective scavenger of hydroxyl, peroxyl and superoxide radicals in both the aqueous and lipid phases. The commonly used chemotherapeutic drug BLM-induced pulmonary injury involves generation of oxidant species by an iron-dependent mechanism (11). Further damage is elicited by increased amounts of reactive oxygen species (ROS) produced by activated inflammatory cells which accumulate in the lung lesions induced by BLM (30). However, the direct linkage of ROS formation and PF has not been firmly established.

The results of the present study show that antioxidant LA was effective in reducing lung injury by intratracheal instillation of BLM in rats. The mechanism through which LA limits PF remains unclear but, since it is a cell-permeable compound, it readily enters cells and may act directly as an oxygen radical scavenger. Also, the observed beneficial effect of LA is associated with its antioxidant effect as well as diminished accumulation of collagen and the increment of the MMP-1/TIMP-1 ratio. Previously, we reported that induction of activation of nuclear factor- $\mathrm{\kappa B}(\mathrm{NF}-\kappa \mathrm{B})$, a transcription factor that regulates $>200$ genes, was correlated with cellular toxicity induced by ionizing radiation (31) and with radiation-induced adaptive responses $(32,33)$. Because BLMinduced ROS generation is due to NF- $\mathrm{KB}$ activation (34), and antioxidant (e.g. NAC) inhibits NF-кB activation $(35,36)$, we speculate that LA might inhibit NF- $\mathrm{B}$ activity to reduce lung injury. The possible mechanism of LA/NF-кB-mediated protection of BLM-induced PF needs to be clarified.

Earlier reports suggest that lung damage by BLM appears to act via the generation of ROS, which participates in DNA chain breakage (37) and lipid peroxidation of the cell membrane (38). To determine the antioxidant effect of LA on BLM-induced lung injury, we measured MDA (malondialdehyde), the GSH/GSSG ratio and T-AOC (total antioxidant capacity). Our data showed that MDA, a key marker of lipid peroxidation, was increased in BLM-treated rats at day 14 and 28, but decreased in the BLM+LA group. High levels of reduced glutathione (GSH), an important antioxidant defense mechanism in lungs, play a critical role in the protection of lung cells against oxidative damage (39). By measuring the levels of GSH and GSSG (oxidized glutathione), we found that the ratio of GSH/GSSG was lower in the BLMtreated rats compared to the control rats at day 14 or 28 , while treatment with LA resulted in a significant enhancement in the GSH/GSSG ratio, which indicates an attenuation of oxidant stress. T-AOC levels, including superoxide dismutase, catalase, vitamin $\mathrm{C}$ and vitamin $\mathrm{E}$, represent the ability and status of the antioxidant system in the lungs. In BLM-treated rats, T-AOC was found reduced at each time point we examined, which could be due to the consumption or inhibition of the synthesis mechanism of antioxidants by ROS. This reduction of T-AOC was significantly reversed in the rats treated with intraperitoneal LA. This result, collectively with those of the MDA content and GSH/GSSG ratio, indicate the attenuation of BLM-produced oxidant stress by LA. Also, previous studies suggest that several natural or synthetic antioxidants are protective in BLMinduced PF (40-43).

The fibrotic response, an irreversible process, is characterized by a striking increase in fibroblast population and a profound and complex change in extracellular matrix (ECM) turnover. An imbalance between the synthesis and degradation of ECM molecules in the local lung microenvironment appears to be of central importance in the pathogenesis of the fibrotic component of PF. MMPs are key enzymes that regulate tissue remodeling through turnover of ECM in both the normal and pathological conditions. They play a crucial role in the fibrogenic process, as demonstrated through the marked reduction of BLM-induced PF in mice by MMP-inhibitor batimastat (16). Of the 17 members of the MMP family, only three interstitial collagenases, i.e. MMP$1,-8$ and -13 , can catalyze the rate-limiting initiating step in the degradation of the interstitial collagens. Thus, changes in the levels or activities of these MMPs may play a significant role in the altered collagen metabolism of PF. The mechanisms that regulate MMP activity in response to lung injury are largely undefined. Members of the TIMP gene family regulate the actions of MMPs in the injured lung. Furthermore, the altered expression of TIMPs in the pulmonary microenvironment could have important biologic effects independent of their ability to inactivate MMPs. A reduced molar MMP/TIMP ratio is considered to be a hallmark of PF. In humans, increased levels of TIMP mRNA and protein are observed in lungs of patients with IPF, and TIMP expression there exceeds that of MMP (5). TIMP-1 expression and protein 
were also increased by BLM administration $(16,20,44)$. In this study, both MMP-1 mRNA and protein were increased at day 7 post-BLM, but were decreased at day 14 or 28 . On the otherhand, BLM exposure led to a significant increase in TIMP-1 at all time points, while LA lowered its expression at day 14 or 28 . Together with the data of oxidant stress markers, these results suggest that ROS may be involved in the modulation of MMP/TIMP balance.

In conclusion, LA is a potential inhibitor of BLM-induced PF by acting as an antioxidant, as determined by semiquantitative morphological indices of lung injury and lung collagen content (measured as hydroxycholine content and total protein in BALF). Thus, LA may have promise in the prevention of BLM-induced oxidative lung damage and human IPF. However, further studies are needed to investigate the mechanism of the beneficial effect of LA in the treatment of IPF.

\section{Acknowledgements}

The authors are grateful to the graduate and undergraduate students at the Division of Molecular Radiobiology, School of Health, Purdue University, West Lafayette, IN, USA, for their active participation in the data discussion and assistance in the preparation of this manuscript.

\section{References}

1. Ward PA and Hunninghake GW: Lung inflammation and fibrosis. Am J Respir Crit Care Med 157: S123-S129, 1998.

2. Crouch E: Pathobiology of pulmonary fibrosis. Am J Physiol 259: L159-L184, 1990.

3. Strausz J, Muller-Quernheim J, Steppling H, Nagel M and Ferlinz R: Oxygen radical production by alveolar macrophages in sarcoidosis in relation to activity status of bronchoalveolar lavage lymphocytes. Pneumologie 44 (suppl 1): 222-223, 1990.

4. Rahman I, Skwarska E, Henry M, et al: Systemic and pulmonary oxidative stress in idiopathic pulmonary fibrosis. Free Radic Biol Med 27: 60-68, 1999.

5. Braselmann S and McCormick F: Bcr and Raf form a complex in vivo via 14-3-3 proteins. EMBO J 14: 4839-4848, 1995.

6. Katzenstein AL and Myers JL: Idiopathic pulmonary fibrosis: clinical relevance of pathologic classification. Am J Respir Crit Care Med 157: 1301-1315, 1998.

7. Movsas B, Raffin TA, Epstein AH and Link CJ: Pulmonary radiation injury. Chest 111: 1061-1076, 1997.

8. Abratt RP and Morgan GW: Lung toxicity following chest irradiation in patients with lung cancer. Lung Cancer 35: 103-109, 2002.

9. Mehta V: Radiation pneumonitis and pulmonary fibrosis in nonsmall-cell lung cancer: pulmonary function, prediction, and prevention. Int J Radiat Oncol Biol Phys 63: 5-24, 2005.

10. Robbins ME and Zhao W: Chronic oxidative stress and radiation-induced late normal tissue injury: a review. Int $\mathrm{J}$ Radiat Biol 80: 251-259, 2004.

11. Hayman MJ and Enrietto PJ: Cell transformation by the epidermal growth factor receptor and v-erbB. Cancer Cells 3: 302-307, 1991.

12. Yildirim Z, Turkoz Y, Kotuk M, et al: Effects of aminoguanidine and antioxidant erdosteine on bleomycin-induced lung fibrosis in rats. Nitric Oxide 11: 156-165, 2004.

13. Serrano-Mollar A, Closa D, Prats N, et al: In vivo antioxidant treatment protects against bleomycin-induced lung damage in rats. Br J Pharmacol 138: 1037-1048, 2003.

14. Wang HD, Yamaya M, Okinaga S, et al: Bilirubin ameliorates bleomycin-induced pulmonary fibrosis in rats. Am J Respir Crit Care Med 165: 406-411, 2002.

15. Corbel M, Belleguic C, Boichot E and Lagente V: Involvement of gelatinases (MMP-2 and MMP-9) in the development of airway inflammation and pulmonary fibrosis. Cell Biol Toxicol 18: 51-61, 2002.
16. Corbel M, Caulet-Maugendre S, Germain N, Molet S, Lagente V and Boichot E: Inhibition of bleomycin-induced pulmonary fibrosis in mice by the matrix metalloproteinase inhibitor batimastat. J Pathol 193: 538-545, 2001

17. Fukuda Y, Ishizaki M, Kudoh S, Kitaichi M and Yamanaka N: Localization of matrix metalloproteinases-1, -2 , and -9 and tissue inhibitor of metalloproteinase- 2 in interstitial lung diseases. Lab Invest 78: 687-698, 1998.

18. Kolb M, Bonniaud P, Galt T, Sime PJ, Kelly MM, Margetts PJ and Gauldie J: Differences in the fibrogenic response after transfer of active transforming growth factor-betal gene to lungs of 'fibrosis-prone' and 'fibrosis-resistant' mouse strains. Am J Respir Cell Mol Biol 27: 141-150, 2002.

19. Swiderski RE, Dencoff JE, Floerchinger CS, Shapiro SD and Hunninghake GW: Differential expression of extracellular matrix remodeling genes in a murine model of bleomycininduced pulmonary fibrosis. Am J Pathol 152: 821-828, 1998.

20. Madtes DK, Elston AL, Kaback LA and Clark JG: Selective induction of tissue inhibitor of metalloproteinase- 1 in bleomycin-induced pulmonary fibrosis. Am J Respir Cell Mol Biol 24: 599-607, 2001

21. Cortijo J, Cerda-Nicolas M, Serrano A, et al: Attenuation by oral $\mathrm{N}$-acetylcysteine of bleomycin-induced lung injury in rats. Eur Respir J 17: 1228-1235, 2001.

22. Nagase H and Woessner JF Jr: Matrix metalloproteinases. J Biol Chem 274: 21491-21494, 1999.

23. Yagi K: A simple fluorometric assay for lipoperoxide in blood plasma. Biochem Med 15: 212-216, 1976.

24. Brown AC and Lutton JD: The significance of free radicals and free radical scavengers in L1210 leukemia. Adv Exp Med Biol 241: 135-148, 1988.

25. McNeil TL and Beck LV: Fluorometric estimation of GSHOPT. Anal Biochem 22: 431-441, 1968.

26. Suga M, Iyonaga K, Okamoto T, Gushima Y, Miyakawa H, Akaike T and Ando M: Characteristic elevation of matrix metalloproteinase activity in idiopathic interstitial pneumonias. Am J Respir Crit Care Med 162: 1949-1956, 2000.

27. Yoshimura S, Nishimura Y, Nishiuma T, Yamashita T, Kobayashi $\mathrm{K}$ and Yokoyama M: Overexpression of nitric oxide synthase by the endothelium attenuates bleomycin-induced lung fibrosis and impairs MMP-9/TIMP-1 balance. Respirology 11: 546-556, 2006.

28. Gauldie J, Sime PJ, Xing Z, Marr B and Tremblay GM: Transforming growth factor-beta gene transfer to the lung induces myofibroblast presence and pulmonary fibrosis. Curr Top Pathol 93: 35-45, 1999.

29. Lasky JA and Brody AR: Interstitial fibrosis and growth factors. Environ Health Perspect 108 (suppl 4): 751-762, 2000.

30. Rahman I and MacNee W: Regulation of redox glutathione levels and gene transcription in lung inflammation: therapeutic approaches. Free Radic Biol Med 28: 1405-1420, 2000.

31. Wang T, Hu YC, Dong S, et al: Co-activation of ERK, NFkappaB, and GADD45beta in response to ionizing radiation. $\mathrm{J}$ Biol Chem 280: 12593-12601, 2005.

32. Ahmed KM, Dong S, Fan M and Li JJ: Nuclear factor\{kappa\}B p65 inhibits mitogen-activated protein kinase signaling pathway in radioresistant breast cancer cells. Mol Cancer Res 4: 945-955, 2006.

33. Guo G, Yan-Sanders Y, Lyn-Cook BD, et al: Manganese superoxide dismutase-mediated gene expression in radiationinduced adaptive responses. Mol Cell Biol 23: 2362-2378, 2003.

34. Barnes PJ and Adcock IM: NF-kappa B: a pivotal role in asthma and a new target for therapy. Trends Pharmacol Sci 18: 46-50, 1997.

35. Schreck R, Rieber P and Baeuerle PA: Reactive oxygen intermediates as apparently widely used messengers in the activation of the NF-kappa B transcription factor and HIV-1. EMBO J 10: 2247-2258, 1991.

36. Tozawa K: Activation of nuclear factor-kappa B and control of the expression of cell adhesion molecules in human prostate cancer cells. Nippon Hinyokika Gakkai Zasshi 87: 1082-1091, 1996.

37. Trush MA, Mimnaugh EG, Ginsburg E and Gram TE: Studies on the interaction of bleomycin $\mathrm{A} 2$ with rat lung microsomes. II. Involvement of adventitious iron and reactive oxygen in bleomycin-mediated DNA chain breakage. J Pharmacol Exp Ther 221: 159-165, 1982

38. Arslan SO, Zerin M, Vural $\mathrm{H}$ and Coskun A: The effect of melatonin on bleomycin-induced pulmonary fibrosis in rats. J Pineal Res 32: 21-25, 2002. 
39. Giri SN, Hyde DM and Schiedt MJ: Effects of repeated administration of N-acetyl-L-cysteine on sulfhydryl levels of different tissues and bleomycin-induced lung fibrosis in hamsters. J Lab Clin Med 111: 715-724, 1988.

40. Aruoma OI, Halliwell B, Aeschbach R and Loligers J: Antioxidant and pro-oxidant properties of active rosemary constituents: carnosol and carnosic acid. Xenobiotica 22: 257-268, 1992.

41. Ikezaki S, Nishikawa A, Enami T, et al: Inhibitory effects of the dietary antioxidants butylated hydroxyanisole and butylated hydroxytoluene on bronchioloalveolar cell proliferation during the bleomycin-induced pulmonary fibrosing process in hamsters. Food Chem Toxicol 34: 327-335, 1996.
42. Ledwozyw A: Protective effect of liposome-entrapped superoxide dismutase and catalase on bleomycin-induced lung injury in rats. I. Antioxidant enzyme activities and lipid peroxidation. Acta Vet Hung 39: 215-224, 1991.

43. Venkatesan N, Punithavathi V and Chandrakasan G: Curcumin protects bleomycin-induced lung injury in rats. Life Sci 61: PL51-PL58, 1997.

44. Manoury B, Nenan S, Leclerc O, et al: The absence of reactive oxygen species production protects mice against bleomycininduced pulmonary fibrosis. Respir Res 6: 11, 2005. 